\title{
Electron Diffraction from a Single Atom and Optimal Signal Detection
}

\author{
David A. Muller ${ }^{1,2}$, Yimo Han ${ }^{1}$, Kayla X. Nguyen ${ }^{3}$, Mark W. Tate ${ }^{4}$, Prafull Purohit ${ }^{4}$, Saien Xie ${ }^{1}$, \\ Jiwoong Park ${ }^{3}$, Sol M. Gruner ${ }^{4}$ \\ 1. School of Applied and Engineering Physics, Cornell University, Ithaca, NY, USA \\ 2. Kavli Institute for Nanoscale Science, Cornell University \\ 3. Department of Chemistry and Chemical Biology, Cornell University, Ithaca, NY, USA \\ 4. Department of Physics, Cornell University, Ithaca, NY, USA
}

Imaging single atoms against a supporting background, made of many more atoms, is a common challenge for many areas of materials characterization - from detecting dopants in semiconductors $[1,2]$ to understanding the behavior of catalysts [3]. Electron energy loss spectroscopy and x-ray analysis can help to suppress the background support. While single atom detection is possible [2], their low cross sections imply they are not dose efficient or well-suited to radiation-sensitive materials. The elastic signal is the largest atomic-resolution signal available, but efficient discrimination of the target atom against the support atoms can be system specific. In the best case of single atoms on a graphene or uniform 2D support, the problem is well posed, and the optimal single-channel STEM detector is a low-angle annular dark field (ADF) detector [4]. The inclusion of a thick support considerably complicates matters, but detection of heavy atoms against a light matrix is possible using high-angle ADF imaging [1-3]. The question is can we do better by recording the full scattering distribution?

To compare all possible elastic imaging modes simultaneously we employ a new type of detector that we have recently developed, an electron microscope pixel array detector (EMPAD) that functions as a compact and highspeed, high dynamic range electron diffraction camera (Figure 1a). It has single electron sensitivity with a signal/noise ratio of 140 for a single electron at $200 \mathrm{keV}$ [5]. It has a dynamic range of $10^{6}$ for primary electrons- i.e., a pixel can detect from 1 to 1,000,000 electrons, and reads out an image frame in $0.86 \mathrm{~ms}$. These properties allow us to record essentially an image of all the transmitted electrons, from the unscattered beam to out beyond the HOLZ lines, and do so for every probe position in a real-space, atomic resolution image.

The detector allowed us to reconstruct all possible combinations of single channel images on an absolute and quantitative scale. Here we study stable model systems for both isolated single atoms, and single atoms on a support: 2D materials that are both free-standing, and placed over a finite support of $10 \mathrm{~nm}$ of silicon nitride. Fig. 1 shows such signals extracted from the same 4D-STEM EMPAD data set recorded at atomic

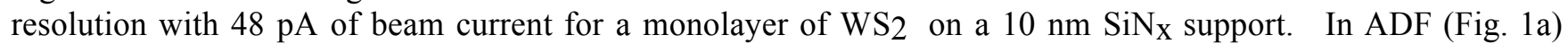

the $\mathrm{W}$ atoms are readily resolved and detected above the amorphous background. The $2^{\text {nd }}$ moment image, calculated from the mean squared angular spread of the diffraction pattern (Fig. 1c) also shows the W clearly. Bright field (Fig. 1b) and annular bright field (Fig. 1d) fail to show the W atoms at all. They are instead dominated by the contrast from the amorphous support. Fig. 2 shows electron diffraction patterns extracted from a single pixel on (Fig. 2b) and away from single W atoms (Fig. 2c). The central disk, where all contrast changes in the weak phase approximation would be contained is dominated by scattering from the thick substrate. Thus methods such as ptychography and unweighted principal components analysis will be dominated by substrate contrast as well. Instead considerable changes in the angular distribution outside the central disk are apparent even for the single heavy atom, explaining the contrast change for the $2^{\text {nd }}$ moment and ADF images [6].

References:

[1] P.M. Voyles et al, Nature 416 (2002), 826 
[2] U. Kaiser, et al, Nature Materials 1 (2002), 102-105

[3] M. M. J. Treacy and S. B. Rice. Journal of Microscopy-Oxford 156 (1989), 211-234

[4] R. Hovden and D. A. Muller. Ultramicroscopy 123 (2012), 59-65

[5] M. W. Tate, et al, Microsc. Microanal. 22, 237-249 (2016).

[6] EM development supported by the NSF MRSEC program (DMR 1120296). Detector development supported by DOE DE-FG02-10ER46693 and the Kavli Institute at Cornell.
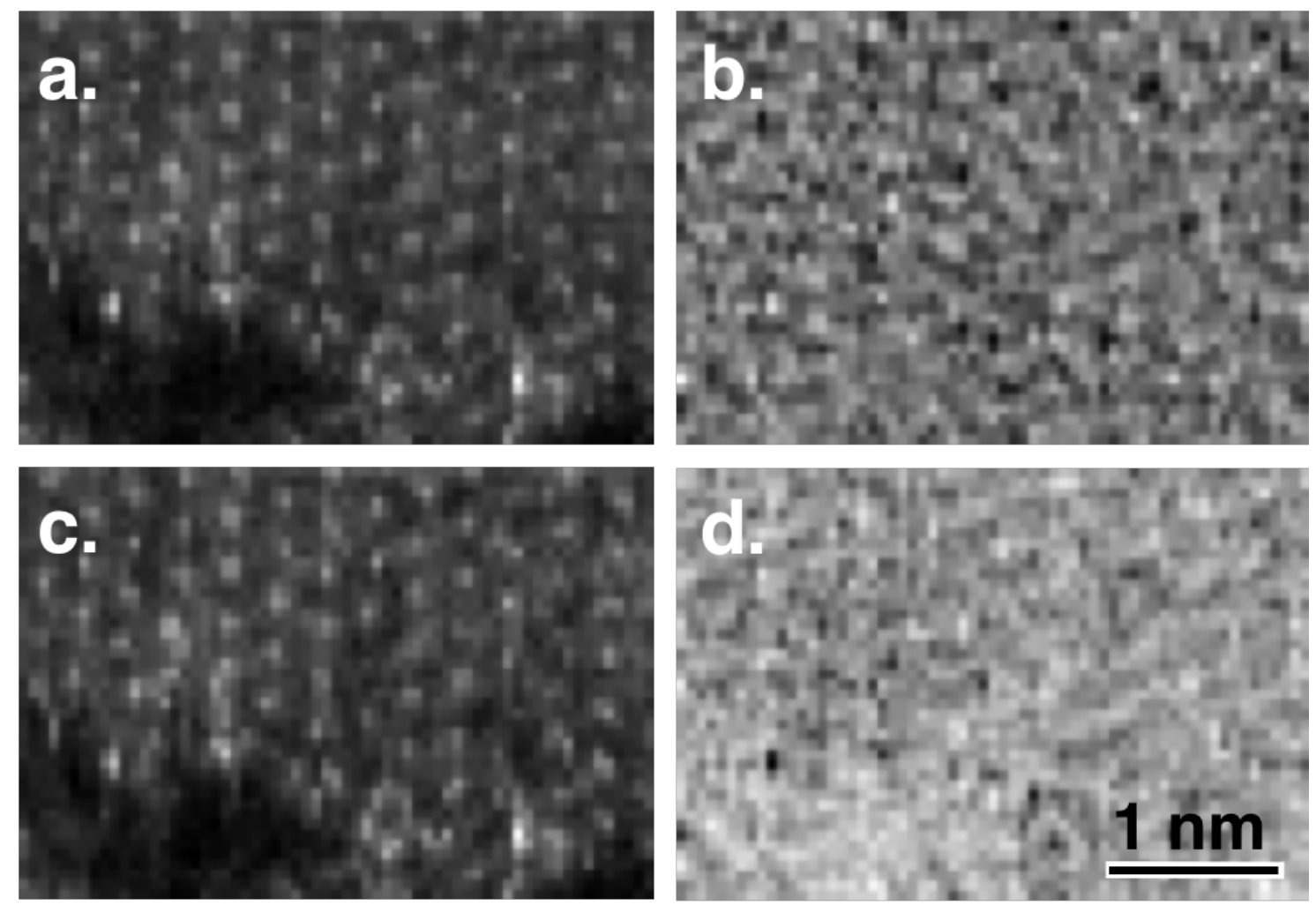

Figure 1. EMPAD 4D-images recorded from a Monolayer $\mathrm{WS}_{2}$ on a $10 \mathrm{~nm}$ silicon nitride support with a $30 \mathrm{mrad}$ convergence semiangle at $120 \mathrm{keV}$. Simultaneously extracted a) ADF (50-120 mrad), b) BF (0-6 mrad), c) 2nd Moment and d) ABF (15-33 mrad) images. Information in the central disk $(b, d)$ is dominated by the amorphous support. High angle scattering is sensitive to the $\mathrm{W}$ atoms $(\mathrm{a}, \mathrm{c})$.
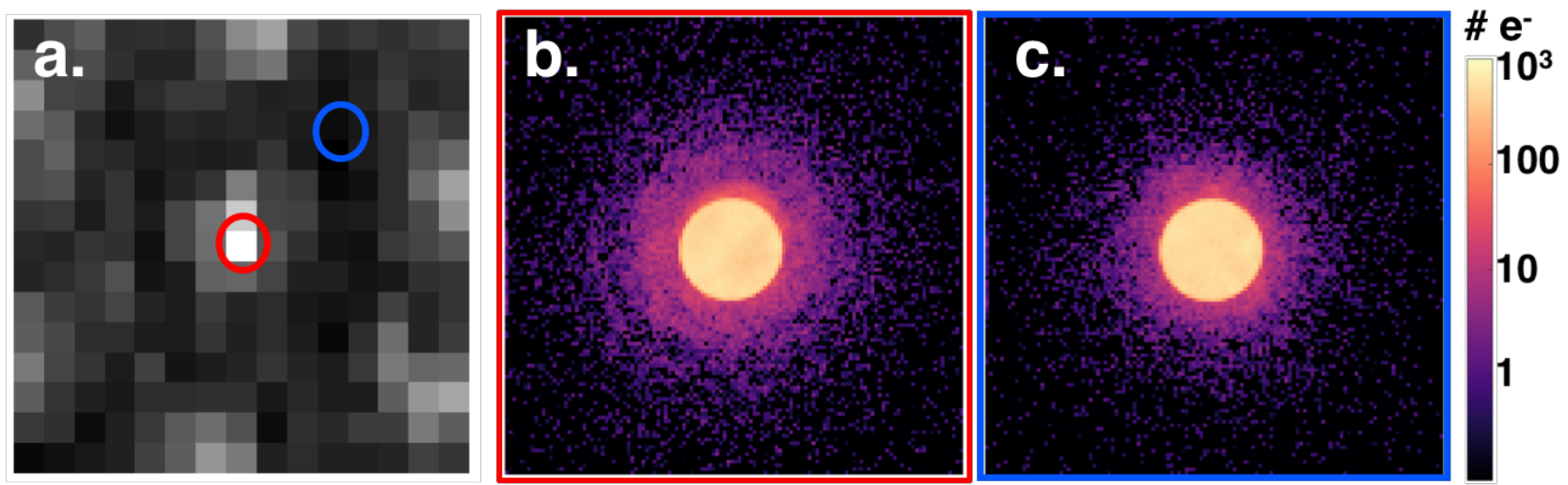

Figure 2. CBED patterns extract from a $\mathrm{W}$ atom (red circle, panel b.) and away from $\mathrm{W}$ atom (blue circle, panel c). This is a subset of Fig 1. Central disk contrast is dominated by the support, but a marked increase in the higher angle scattering is visible for the on-atom diffraction pattern w.r.t. to off-atom. 